\title{
Screening for Humoral Immunodeficiency in Patients with Community-Acquired Pneumonia
}

\author{
Karthik Vadamalai, MD'; Denise Sanchez-Tejera, MD'; Jonathan Bress, MD ${ }^{1,2}$; S Shahzad Mustafa, MD1,3*
}

${ }^{1}$ Rochester Regional Health, Rochester, New York; ${ }^{2}$ Rochester Institute of Technology, Rochester, New York; ${ }^{3}$ University of Rochester School of Medicine and Dentistry, Rochester, New York.

BACKGROUND: Immunodeficiency is an underrecognized risk factor for infections, such as community-acquired pneumonia (CAP).

OBJECTIVE: We evaluated patients admitted with CAP for humoral immunodeficiency.

DESIGN: Prospective cohort study

SETTING: Inpatients

PATIENTS, INTERVENTION, AND MEASUREMENTS: We enrolled 100 consecutive patients admitted with a diagnosis of CAP from February 2017 to April 2017. Serum lgG, IgM, $\lg A$, and lgE levels were obtained within the first 24 hours of admission. CURB-65 score and length of hospital stay were calculated. The Wilcoxon rank-sum test, Kruskal-Wallis test, and simple linear regression analysis were used in data analysis.

RESULTS: The prevalence of hypogammaglobinemia in patients with CAP was $38 \%$ ( $95 \% \mathrm{Cl}: 28.47 \%$ to 48.25\%). Twenty-seven of 100 patients had lgG hypogammaglobinemia (median: $598 \mathrm{mg} / \mathrm{dL}$, IQ range: 459-654), 23 of 100 had IgM hypogammaglobinemia (median: $38 \mathrm{mg} / \mathrm{dL}$, IQ range: $25-43$ ), and 6 of 100 had IgA hypogammaglobinemia (median: $36 \mathrm{mg} / \mathrm{dL}$, $\mathrm{Q}$ range: 18-50). The median hospital length of stay for patients with IgG hypogammaglobinemia was significantly higher when compared to patients with normal lgG levels (five days, IQ range [3-10] vs three days, 10 range [2-5], $P=$ .0085). Fourteen patients underwent further immune evaluation, resulting in one diagnosis of multiple myeloma, three patients diagnosed with specific antibody deficiency, and one patient diagnosed with selective IgA deficiency.

CONCLUSION: There is a high prevalence of hypogammaglobinemia in patients hospitalized with CAP, with IgG and IgM being the most commonly affected classes. IgG hypogammaglobinemia was associated with an increased length of hospitalization. Screening immunoglobulin levels in CAP patients may also uncover underlying humoral immunodeficiency or immunoproliferative disorders. Journal of Hospital Medicine 2019;14:33-37. Published online first November 28, 2018. (C) 2019 Society of Hospital Medicine ommunity-acquired pneumonia (CAP) is the most common infection in hospitalized patients and the eighth most common cause of death in the United States. ${ }^{1}$ Mortality from CAP is estimated to be $5.1 \%$ in the outpatient population, $13.6 \%$ in hospitalized patients, and $35.1 \%$ in patients admitted to the intensive care unit. ${ }^{2,3}$ CAP accounts for more than 50,000 deaths annually in the United States. ${ }^{2}$ There are multiple risk factors for CAP, including tobacco use, malnutrition, chronic obstructive pulmonary disease (COPD), bronchiectasis, cystic fibrosis, and mechanical bronchial obstruction. Underlying immunodeficiency, specifically humoral immunodeficiency, is also a risk factor for CAP.

Primary immunodeficiency (PIDD) is estimated to affect one in 1,800 individuals in the United States. ${ }^{4}$ The National Insti-

*Corresponding Author: S. Shahzad Mustafa, MD; Telephone: 585-922-8350;
E-mail: shahzad.mustafa@rochesterregional.org

Received: June 13, 2018; Revised: September 29, 2018;

Accepted: October 2, 2018

@ 2019 Society of Hospital Medicine DOI 10.12788/jhm.3106 tutes of Health (NIH) estimates that only one out of three individuals with PIDD are appropriately diagnosed. Based on probability calculations on known PIDD patients versus incidence of disease, the NIH estimates that more than 500,000 individuals with PIDD remain undiagnosed in the United States. ${ }^{4}$ Further, there exists an average diagnostic delay of at least five years. This delay increases both morbidity and mortality and leads to increased healthcare utilization. 5,6

The most common form of primary immunodeficiency is due to humoral immunodeficiency, including selective IgA deficiency, specific antibody deficiency, and common variable immunodeficiency. Specific antibody deficiency is defined as a lack of response to polysaccharide antigens in the setting of low to normal Ig levels and an intact response to peptide antigens. ${ }^{7}$ Selective IgA deficiency is defined as the isolated deficiency of serum IgA in the setting of normal serum levels of IgG and IgM in an individual older than four years in whom other causes of hypogammaglobinemia have been excluded. ${ }^{8}$ Common variable immunodeficiency (CVID) is defined as a decreased serum concentration of IgG in combination with low levels of IgA and/or IgM with a poor or absent response to immunization in the absence of other defined immunodeficiency 
state. ${ }^{9}$ In addition to experiencing recurrent infections-namely bronchitis, sinusitis, otitis, and pneumonia-patients with CVID are also at increased risk of autoimmunity and malignancy. In adults, secondary immunodeficiency is more common than primary immunodeficiency. Secondary immunodeficiency occurs commonly with disease states like HIV infection, diabetes, cirrhosis, malnutrition, and autoimmune conditions. ${ }^{10} \mathrm{Ad}$ ditional causes of secondary immune defects due to humoral immunodeficiency include immune-modulating drugs-such as rituximab and ibrutinib-and hematologic malignancies, including chronic lymphocytic leukemia and multiple myeloma. Recurrent infections remain the leading cause of morbidity and mortality in patients with both primary and secondary immunodeficiency. ${ }^{11,12}$

Evaluation of the humoral immune system begins with measurement of serum immunoglobulin (lg) levels. Although abnormal Ig levels are not diagnostic of immunodeficiency, abnormal results may prompt additional evaluation. Screening strategies may assist in making an earlier diagnoses, potentially decreasing morbidity and mortality in patients with immunodeficiency. ${ }^{13-15}$ To date, there have been no studies evaluating the utility of screening Ig levels to evaluate for underlying humoral immunodeficiency in patients hospitalized for CAP.

\section{METHODS}

\section{Study Design}

This was a prospective cohort study conducted at Rochester General Hospital, a 528-bed tertiary care medical center, from February 2017 to April 2017. We enrolled 100 consecutive patients admitted to the inpatient internal medicine service with a physician diagnosis of CAP. Written consent was obtained from each patient. The study was approved by the institutional review board at Rochester General Hospital.

\section{Case Definition}

The following criteria were used to diagnose CAP: (1) Respiratory symptoms of productive cough or pleuritic chest pain, (2) Fever $>38^{\circ} \mathrm{C}$ before or at the time of admission, and (3) chest imaging with infiltrate. Exclusion criteria included a diagnosis of hospital-acquired pneumonia, prior diagnosis of primary immunodeficiency, immunosuppression due to an underlying condition, such as HIV or malignancy, therapy with immunosuppressive medications including chemotherapy, Ig replacement within the past six months, or treatment with $>10 \mathrm{mg}$ prednisone for greater than 14 days before hospital admission.

Patients underwent an additional evaluation by a clinical immunologist if they met one of the following criteria: any hypergammaglobinemia (elevated IgG, IgM, or IgA), IgG hypogammaglobinemia $<550 \mathrm{mg} / \mathrm{dL}$, undetectable $\operatorname{lgM}$ or $\lg \mathrm{A}$ or if lgG, IgM, and IgA were all below the lower limit of normal.

CURB-65 was used for estimation of the severity of illness with CAP. The components of the score include age $\geq 65$, confusion, BUN $>19 \mathrm{mg} / \mathrm{dl}$, respiratory rate $\geq 30$ breaths per minute and systolic blood pressure $<90 \mathrm{~mm} \mathrm{Hg}$ or diastolic blood pressure $\leq 60 \mathrm{~mm} \mathrm{Hg}$. Each component is scored zero if absent or one if present. Predicted mortality ranges from $0.6 \%$ for a score of zero to $27.8 \%$ for a score of 5 .

\section{Data Collection}

Patient health information including age, race, gender, medical history, admission notes, results of chest imaging studies, and relevant laboratory studies including serum levels of IgG, IgM, IgA, IgE on admission was obtained from the electronic medical health record. An additional evaluation by the immunologist occurred within three months of hospital discharge and included repeat Ig levels, pre- and postvaccination titers of polysaccharide and peptide antigens, serum protein electrophoresis, and B \& T cell panels.

\section{Description of Normal Levels}

The normal levels of immunoglobulins were defined based on standard reference ranges at the laboratory at Rochester General Hospital; IgG (700-1,600 mg/dl), IgM (50-300 mg/dl), IgA (70-400 mg/dl), and lgE $(0-378 \mathrm{lU} / \mathrm{ml})$. Although there is no established classification regarding the degree of IgG hypogammaglobinemia, ${ }^{16}$ clinical immunologists commonly classify the severity of IgG hypogammaglobinemia as follows: mild (550$699 \mathrm{mg} / \mathrm{dL})$, moderate (400-549 mg/dL), and severe (<400 mg/ dL) lgG hypogammaglobinemia.

\section{Statistical Analysis}

Statistical analysis was performed using STATA software (StataCorp LLC, College Station, Texas). We conducted a Wilcoxon rank-sum test to compare the median difference in length of stay between groups with a low versus normal range of immunoglobulins. A Kruskal-Wallis test was performed to check for the median difference in IgG levels across degrees of illness severity (CURB-65 score categories). We conducted a simple linear regression analysis using the logarithmic data of the length of stay and lgG level variables. A chi-square test was used to determine the association between comorbidities and Ig levels.

\section{RESULTS}

\section{Baseline Characteristics}

There were 100 patients with CAP enrolled in this study with a median age of $65.04 \pm 18.8$, and 53\% were female. Forty-seven patients reported a previous history of pneumonia and 18 reported a history of recurrent sinusitis or otitis media. Of the 100 enrolled patients, 46 had received pneumococcal polysaccharide vaccine (PPV23), 26 had received the 13-valent pneumococcal conjugate vaccine (PCV13), and 22 had received both (Table 1). The mean white blood cell count on admission was $12.9 \pm 7 \times 10^{3} / \mathrm{uL}$ with $75 \pm 12.5 \%$ neutrophils. Total protein $(6.5$ $\pm 0.8)$ and albumin $(3.7 \pm 0.5)$ were within the normal range for the study population.

\section{Immunoglobulin Analyses}

The prevalence of hypogammaglobinemia in the study was $38 \%$ (95\% Cl: $28.47 \%$ to $48.25 \%$ ). The median values of $\mathrm{lg}$ levels 
for the entire study population and in patients with hypogammaglobinemia are summarized in Table 2.

- IgG hypogammaglobinemia (<700 mg/dl) was found in 27/100 patients, with a median level of $598 \mathrm{mg} / \mathrm{dL}$, IQ range: 459-654. The median age in this group was 76.5 years, and 13 were female. Of these 27 patients, 10 had low lgM, four had low IgA, and four had an elevated IgE. In this group, 11 patients had received PPSV23, nine had received PCV13, and six had received both PPV23 and PCV13 before the index hospital admission.

- IgG hypergammaglobinemia (>1,600 mg/dl) was found in $9 / 100$ patients, with a median level of $1,381 \mathrm{mg} / \mathrm{dL}, \mathrm{IO}$ range: 1,237-1,627. The median age was 61 years, and six were female. Of these nine patients, three had low lgM, one had low IgA, and four had elevated lgE.

- IgM hypogammaglobinemia (<50 mg/dl) was found in 23/100 patients with a median level of $38 \mathrm{mg} / \mathrm{dL}, \mathrm{IQ}$ range: 25-43. In this group, the median age was 69 years, and 10 were female. Of these 23 patients, 10 had low lgG, and three had an elevated IgG.

- IgM hypergammaglobinemia (>300 mg/dl) was noted in two patients, with a median level of $491 \mathrm{mg} / \mathrm{dL}$, IQ range: 418 564. Both patients were female, and one had elevated lgG.

- IgA hypogammaglobinemia (<70 mg/dl) was discovered in six patients, with a median level of $36 \mathrm{mg} / \mathrm{dL}$, IQ range: 18 50. In this group, four patients had low lgG, four had low IgM, one had elevated IgE, and one had elevated lgG.

- IgA hypergammaglobinemia (>400 mg/dl) was noted in five patients, with a median level of $561 \mathrm{mg} / \mathrm{dL}$, IO range: 442565: Two patients were female. Of these five patients, one had high lgG, and one had low lgG.

\section{Length of Stay and Severity of Pneumonia}

The median length of stay in the hospital for the entire study population was three days (IQ range: 2-5.5 days). Among patients with lgG hypogammaglobinemia, the median length of stay was two days longer as compared with patients who had IgG levels in the normal range (5 days, IQ range [3-10] vs 3days, IQ range [2-5], $P=.0085$ ).

The median CURB-65 score for the entire study population was two (IQ range: 1-3). The median CURB-65 score did not differ between patients with low and normal ranges of lgG lev-
TABLE1. Characteristics of Patients

\begin{tabular}{|c|c|}
\hline Total participants (n) & 100 \\
\hline Age- years & $65.04 \pm 18.71$ \\
\hline Female-no (\%) & $53(53 \%)$ \\
\hline \multicolumn{2}{|l|}{ Race-no (\%) } \\
\hline White & $67(67 \%)$ \\
\hline African-American & $20(20 \%)$ \\
\hline Hispanic & $10(10 \%)$ \\
\hline Asian & $2(2 \%)$ \\
\hline Indian & $1(1 \%)$ \\
\hline \multicolumn{2}{|l|}{ Medical History—no (\%) } \\
\hline COPD & $25(25 \%)$ \\
\hline Asthma & $29(29 \%)$ \\
\hline Bronchiectasis & $1(1 \%)$ \\
\hline Obesity (BMI $\geq 30$ ) & $38(38 \%)$ \\
\hline Diabetes & $27(27 \%)$ \\
\hline History of physician-diagnosed pneumonia & $47(47 \%)$ \\
\hline History of recurrent sinusitis/otitis & $18(18 \%)$ \\
\hline Current smoker & $38(38 \%)$ \\
\hline \multicolumn{2}{|l|}{ Vaccination Status-no (\%) } \\
\hline PPV13 vaccination & $26(26 \%)$ \\
\hline PPV23 vaccination & $46(46 \%)$ \\
\hline History of PPSV 23 + PPV 13 vaccination & $22(22 \%)$ \\
\hline \multicolumn{2}{|l|}{ CURB65-no (\%) } \\
\hline 0 & $24(24 \%)$ \\
\hline 1 & $25(25 \%)$ \\
\hline 2 & $24(24 \%)$ \\
\hline 3 & $20(20 \%)$ \\
\hline 4 & $7(7 \%)$ \\
\hline
\end{tabular}

els (Median: 2, IQ range [1-3] vs Median: 1 , IQ range [0-3], $P=$ .2922). The CURB-65 score was not correlated with lgG levels $(\rho=-0.0776, P=.4428)$. Length of stay, however, was positively correlated with CURB-65 score $(\rho=.4673, P=.000)$

A simple linear regression analysis using the logarithmic transformation of both length of stay and IgG level revealed a linear relationship between serum lgG levels and hospital length of stay $\left(P=.0335,\left[R^{2}=.0453\right]\right)$.

TABLE 2. Serum Immunoglobulin Levels in the Study Population

\begin{tabular}{|c|c|c|c|}
\hline Type & Total Number of Patients & Median (mg/dl) & IQ Range in $\mathrm{mg} / \mathrm{dl}$ \\
\hline $\lg G(700-1,600 \mathrm{mg} / \mathrm{dl}))$ & 100 & 941 & $684.5-1223$ \\
\hline Low lgG ( <700 mg/dl) & 27 & 598 & $459-654$ \\
\hline $\lg A(70-400$ mg/dl) & 100 & 228 & $164-292.5$ \\
\hline Low IgA (<70 mg/dl) & 6 & 36 & $18-50$ \\
\hline $\operatorname{lgM}(50-300$ mg/dl) & 100 & 76.5 & $52-114$ \\
\hline Low IgM (<50 mg/dl) & 23 & 38 & $25-43$ \\
\hline
\end{tabular}


TABLE 3. Serum Immunoglobulin Levels in Patients with Diagnosis of Antibody Deficiency

\begin{tabular}{|c|c|c|c|c|}
\hline Age in Years & $\lg G(700-1,600)$ & $\operatorname{lgM}(50-300 \mathrm{mg} / \mathrm{dl})$ & $\lg A(70-400 \mathrm{mg} / \mathrm{dl})$ & Diagnosis \\
\hline 79 & 1,904 & 8 & 18 & Multiple myeloma \\
\hline 77 & 359 & 23 & 30 & Selective antibody deficiency \\
\hline 54 & 680 & 24 & 42 & Selective antibody deficiency \\
\hline 90 & 337 & 79 & 77 & Selective antibody deficiency \\
\hline 56 & 1,321 & 100 & $<18$ & Selective IgA deficiency \\
\hline
\end{tabular}

\section{Comorbidities and New Diagnoses}

No significant association was found between smoking status, obesity, COPD, asthma, diabetes mellitus, and hypogammaglobinemia.

Fourteen patients with abnormal Ig levels as defined by (1) the presence of hypergammaglobinemia (elevated IgG, IgM, or IgA), (2) IgG levels <550, (3) undetectable IgA or IgM, and (4) either IgG or both IgM and IgA below the lower limit of normal underwent further evaluation. Of these 14 patients, one was diagnosed with multiple myeloma, one with selective IgA deficiency, and three with specific antibody deficiency (Table 3).

\section{DISCUSSION}

Previous research has evaluated the humoral immune system during an episode of CAP. ${ }^{17-20}$ Studies on lg levels in patients with CAP have shown hypogammaglobinemia to be associated with ICU admission and increased ICU mortality. ${ }^{17,20}$ Additionally, patients with CAP have been shown to have lower $\operatorname{lgG}_{2}$ levels than healthy controls. The goal of our study was to evaluate patients with CAP for humoral immunodeficiency.

In our study, the prevalence of low Ig levels in CAP was 38\%, with IgG hypogammaglobinemia being the most common class of hypogammaglobinemia. This rate is slightly higher than that found in a previous work by de la Torri et al., ${ }^{21}$ who reported a prevalence of $28.9 \%$ in the inpatient population. The lower prevalence in the de la Torri et al. study was likely secondary to the exclusion of patients who did not have recorded Ig levels. ${ }^{21}$ Additionally, de la Torri et al. noted an inverse relationship between serum IgG levels and CURB-65. These results were not replicated in our analysis. This is likely due to the relatively low number of patients in each category of CURB-65 score in our study focusing only on inpatients. However, low IgG levels were associated with increased length of stay (5 days, IO range [3-10] vs 3 days, 10 range [2-5]).

Sepsis can cause hypogammaglobinemia. ${ }^{22,23}$ The mechanism behind this phenomenon remains unclear, but several theories have been proposed. Sepsis results in endothelial dysfunction, vascular leakage, lymphopenia, and quantitative and qualitative defects in T and B cells. ${ }^{23}$ This potentially leads to impaired production and increased catabolism of immunoglobulins. Immunoglobulins play an essential role in recovery from sepsis, and there may be increased consumption during acute illness. ${ }^{24-28}$ Regardless of the mechanism, hypogamma- globinemia with SIRS, sepsis, and septic shock has been shown to be a risk factor for increased mortality in these patients. ${ }^{22,23}$ There is currently no consensus on the optimal time to screen for humoral immunodeficiency or evaluate the immune system after infection, such as CAP. Some would argue that lg levels are lower during an active illness and, therefore, this may not be an appropriate time to evaluate lg levels. However, we believe that inpatient hospitalization for CAP provides a window of opportunity to selectively screen these patients at higher risk for PIDD for underlying immune defects. A hospital-based approach as demonstrated in this study may be more productive than relying on an outpatient evaluation, which often may not occur due to patient recall and/or fragmentation of care, thus leading to the well-recognized delay in diagnosis of immunodeficiency. 5

In our study, one patient was diagnosed with multiple myeloma, three were diagnosed with specific antibody deficiency, and one was diagnosed with selective IgA deficiency. The patient with multiple myeloma was a 79-year old male who presented with his first ever episode of CAP, along with modest anemia and a creatinine of 1.6. His only other infectious history included an episode of sinusitis and one episode of pharyngitis. Additional evaluation included serum and urine electrophoresis, followed by bone marrow biopsy. This patient's multiple myeloma diagnoses may have been missed if lg levels had not been evaluated. Three patients were diagnosed with specific antibody deficiency. All these patients were above 50 years of age; two out of the three patients in this group had experienced a previous episode of pneumonia, and one had a history of recurrent sinusitis. Lastly, one patient was diagnosed with selective IgA deficiency as defined by undetectable IgA in the setting of normal lgG and IgM. This 56-year-old patient had a history of multiple episodes of sinusitis and three previous episodes of pneumonia, one requiring inpatient hospitalization. Earlier diagnosis of patients with specific antibody deficiency and selective IgA deficiency can guide management, which focuses on appropriate vaccination, the use of prophylactic antibiotics, and the possible role of Ig replacement in patients with specific antibody deficiency.

Of the 100 patients who underwent screening for immunodeficiency in the setting of CAP, five were found to have clinically significant humoral immunodeficiency, resulting in a number needed to screen of 20 to detect a clinically meaningful immunodeficiency in the setting of CAP. The number needed 
to screen by colonoscopy to detect one large bowel neoplasm in patients $>50$ years of age is $23 .{ }^{29}$ The number needed to screen to diagnose one occult cancer after an unprovoked DVT is $91 . .^{30}$ Based on this information, we feel that future, larger studies are required to evaluate the utility and cost-effectiveness of routine Ig screening for CAP requiring inpatient hospital admission.

We acknowledge limitations to this study. First, this study only evaluated adults in the inpatient floor setting, and therefore the results cannot be applied to the pediatric population or patients in the outpatient or ICU setting. Second, rather than completing a follow-up evaluation in all patients with abnormal immunoglobulins, we selected patients for additional evaluation based on criteria predefined by an immunologist. Although our rationale was to minimize additional diagnostic testing in individuals with mild hypogammaglobinemia, we acknowledge that this could have led to missing subtler humoral defects, such as a patient with near-normal Ig levels but a suboptimal response to vaccination. Third, due to the design of the study, we did not have a healthy matched control group. Despite these limitations, we believe our results are clinically meaningful and warrant future, larger scale investigation.

In conclusion, there is a high prevalence of hypogammaglobinemia in patients admitted with the diagnosis of CAP. IgG hypogammaglobinemia is the most commonly decreased class of lg, and hospital length of stay is significantly longer in patients with low levels of lgG during admission for CAP. Additional immune evaluation of patients with CAP and abnormal Ig levels may also result in the identification of underlying antibody deficiency or immunoproliferative disorders.

Disclosures: The authors have nothing to disclose

\section{References}

1. File TM, Marrie TJ. Burden of community-acquired pneumonia in North American adults. Postgrad Med. 2010;122(2):130-141. doi: 10.3810/ pgm.2010.03.2130

2. Solomon CG, Wunderink RG, Waterer GW. Community-acquired pneumonia. N Engl J Med. 2014;370(6):543-551. doi: 10.1056/NEJMcp1214869.

3. Fine MJ, Smith MA, Carson CA, et al. Prognosis and outcomes of patients with community-acquired pneumonia. A meta-analysis. JAMA. 1996;275(2):134-141. doi: 10.1001/jama.1996.03530260048030.

4. Dantas EO, Aranda CS, Nobre FA, et al. The medical awareness concerning primary immunodeficiency diseases (PID) in the city of Sao Paulo, Brazil. J Allergy Clin Immunol. 2012;129(2):AB86. doi: 10.1016/j.jaci.2011.12.648.

5. Kobrynski L, Powell RW, Bowen S. Prevalence and morbidity of primary immunodeficiency diseases, United States 2001-2007. J Clin Immunol. 2014;34(8):954-961. doi: 10.1007/s10875-014-0102-8.

6. Seymour B, Miles J, Haeney M. Primary antibody deficiency and diagnostic delay. J Clin Pathol. 2005;58(5):546-547. doi: 10.1136/jcp.2004.016204.

7. Orange JS, Ballow M, Stiehm ER, et al. Use and interpretation of diagnostic vaccination in primary immunodeficiency: A working group report of the Basic and Clinical Immunology Interest Section of the American Academy of Allergy, Asthma \& Immunology. J Allergy Clin Immunol. 2012;130(3 SUPPL.). doi: 10.1016/j.jaci.2012.07.002
8. Yel L. Selective IgA deficiency. J Clin Immunol. 2010;30(1):10-16. doi: 10.1007/ s10875-009-9357-x.

9. Conley ME, Notarangelo LD, Etzioni A. Diagnostic criteria for primary immunodeficiencies. Representing PAGID (Pan-American Group for Immunodeficiency) and ESID (European Society for Immunodeficiencies). Clin Immunol. 1999:93(3):190-197. doi: 10.1006/clim.1999.4799.

10. Chinen J, Shearer WT. Secondary immunodeficiencies, including HIV infection. J Allergy Clin Immunol. 2010;125(2 Suppl 2):S195-S203. doi: 10.1016/j. jaci.2009.08.040

11. Blimark C, Holmberg E, Mellqvist UH, et al. Multiple myeloma and infections: A population-based study on 9253 multiple myeloma patients. Haematologica. 2015;100(1):107-113. doi: 10.3324/haematol.2014.107714.

12. Strati $P$, Chaffee $K$, Achenbach $S$, et al. Disease progression and complications are the main cause of death in patients with chronic lymphocytic leukemia (CLL) independent of age and comorbidities at diagnosis. Blood. 2015; $126(23): 5265$

13. Holding S, Jolles S. Current screening approaches for antibody deficiency. Curr Opin Allergy Clin Immunol. 2015;15(6):547-555. doi: 10.1097/ ACl.0000000000000222.

14. Azar AE, Ballas ZK. Evaluation of the adult with suspected immunodeficiency. Am J Med. 2007;120(9):764-768. doi: 10.1016/j.amjmed.2006.12.013.

15. Stoop JW, Zegers BJM, Sander PC, Ballieux RE. Serum immunoglobulin levels in healthy children and adults. Clin Exp Immunol. 1969;4(1):101-112.

16. Agarwal $S$, Cunningham-Rundles $C$. Assessment and clinical interpretation of reduced IgG values. Ann Allergy Asthma Immunol. 2007;9(3):281-283. doi: 10.1016/S1081-1206(10)60665-5

17. Justel M, Socias L, Almansa R, et al. IgM levels in plasma predict outcome in severe pandemic influenza. J Clin Virol. 2013;58(3):564-567. doi: 10.1016/j. jcv.2013.09.006.

18. Gordon CL, Holmes NE, Grayson ML, et al. Comparison of immunoglobulin $\mathrm{G}$ subclass concentrations in severe community-acquired pneumonia and severe pandemic 2009 influenza A (H1N1) infection. Clin Vaccine Immunol. 2012;19(3):446-448. doi: 10.1128/CVI.05518-11.

19. Gordon $\mathrm{CL}$, Johnson PD, Permezel $\mathrm{M}$, et al. Association between severe pandemic 2009 influenza $A$ (H1N1) virus infection and immunoglobulin $\mathrm{G}(2)$ subclass deficiency. Clin Infect Dis. 2010;50(5):672-678. doi: 10.1086/650462

20. Feldman C, Mahomed AG, Mahida P, et al. IgG subclasses in previously healthy adult patients with acute community-acquired pneumonia. $S$ Afr Med J. 1996;86(5 Suppl):600-602.

21. de la Torre MC, Torán P, Serra-Prat M, et al. Serum levels of immunoglobulins and severity of community-acquired pneumonia. BMJ Open Respir Res. 2016:3(1):e000152. doi:1 0.1136/bmjresp-2016-000152.

22. Prucha M, Zazula R, Herold I, Dostal M, Hyanek T, Bellingan G. Presence of hypogammaglobulinemia in patients with severe sepsis, septic shock, and SIRS is associated with increased mortality. J Infect. 2014;68(3):297-299. doi: 10.1016/j.jinf.2013.11.003.

23. Shankar-Hari M, Culshaw N, Post B, et al. Endogenous IgG hypogammaglobulinaemia in critically ill adults with sepsis: systematic review and meta-analysis. Intensive Care Med. 2015;41(8):1393-1401. doi: 10.1007/s00134015-3845-7.

24. Drewry A, Samra N, Skrupky L, Fuller B, Compton S, Hotchkiss R. Persistent lymphopenia after diagnosis of sepsis predicts mortality. Shock. 2014:42(5):383-391. doi: 10.1097/SHK.0000000000000234.

25. Boomer JS, Shuherk-Shaffer J, Hotchkiss RS, Green JM. A prospective analysis of lymphocyte phenotype and function over the course of acute sepsis. Crit Care. 2012;16(3). doi: 10.1186/cc11404.

26. Nordenfelt $P$, Waldemarson $S$, Linder A, et al. Antibody orientation at bacterial surfaces is related to invasive infection. J Exp Med. 2012;209(13):23672381. doi: 10.1084/jem.20120325.

27. Michaelsen TE, Sandlie I, Bratlie DB, Sandin RH, Ihle O. Structural difference in the complement activation site of human IgG1 and IgG3. Scand J Immunol. 2009;70(6):553-564. doi: 10.1111/j.1365-3083.2009.02338.x

28. Lee WL, Slutsky AS. Sepsis and endothelial permeability. N Engl J Med. 2010;363(7):689-691. doi: 10.1056/NEJMcibr1007320.

29. Regula J, Rupinski M, Kraszewska E, et al. Colonoscopy in colorectal-cancer screening for detection of advanced neoplasia. N Engl J Med. 2006;355(18):1863-1872. doi: 10.1056/NEJMoa054967.

30. Van Es N, Le Gal G, Otten HM, et al. Screening for occult cancer in patients with unprovoked venous thromboembolism. Ann Intern Med. 2017;167(6):410-417. doi: 10.7326/M17-0868 